\title{
Morphological Characterization and Quality Parameters of Various Forage Sorghum Genotypes (Sorghum bicolor L. Moench)
}

\author{
Neha Rohila ${ }^{1 *}$, Satyawan Arya ${ }^{1}$, S. K. Pahuja ${ }^{1}$, Pummy Kumari ${ }^{1}$, \\ Pinki ${ }^{1}$, Kanika Rani ${ }^{2}$ and Nisha Devi ${ }^{2}$ \\ ${ }^{1}$ Department of Genetics and Plant Breeding, COA, CCS Haryana Agricultural University, \\ Hisar-125004, Haryana, India \\ ${ }^{2}$ Department of Molecular Biology, Biotechnology and Bioinformatics, COBSH, CCS \\ Haryana, Agricultural University, Hisar-125004, Haryana, India
}

*Corresponding author

\section{A B S T R A C T}

\begin{tabular}{|l|}
\hline Ke y w or d s \\
$\begin{array}{l}\text { Sorghum, morphological } \\
\text { and quality traits, varietal } \\
\text { characterization }\end{array}$ \\
\hline Article Info \\
\hline $\begin{array}{l}\text { Accepted: } \\
\text { 18 May } 2018 \\
\text { Available Online: } \\
\text { 10 June } 2018\end{array}$ \\
\hline \hline
\end{tabular}

\section{Introduction}

Sorghum (Sorghum bicolor L. Moench) was originated in Africa is one of the five top cereal crops in the world. It is one of the major multi-purpose crops grown for forage and grain production purpose. The yield of sorghum varies from state to state with varying rainfall, soil types and also with varying seasons. It's quick growing habit, high yield, regeneration potential, better palatability, digestibility and drought tolerance makes it good choice of fodder for farming community on which the livestock industry
The present study was conducted on 25 sorghum genotypes grown in Randomized Block Design during Kharif 2015 to evaluate, categorize and classify them for fodder yield and quality traits. Observations were recorded on eighteen variables involving morphological, quality. Out of 25 genotypes, nine genotypes were grouped under yellow green and remaining sixteen genotypes were under greyed purple. Ten genotypes were without stigma anthocyanin colouration and remaining fifteen genotypes had stigma anthocyanin colouration. On the basis of total plant height, stem girth, number of tillers, number of es, green fodder yield and dry fodder yield genotypes were classified and genotype CSV 21F, HJ 260, IS 2205, S 490, S 651, SSG 59-3 showed maximum plant height, number of leaves, number of tillers, stem girth, green fodder yield and dry fodder yield.

depends. It can grow in the areas where all other major cereal crops could not grow successfully (Pholsen and Suksri, 2007).

Descriptors of varieties of crop species are required for characterization of varietal identity, determine varietal purity and establish the distinctiveness of new variety from existing varieties and documentation of genetic resources. In early days, all over the world, a small list of descriptors was sufficient to distinguish between crop varieties in use. However, in the recent decades, the world witnessed the emergence of large and highly competitive variety development programmes, 
particularly in the developed countries and also in some of the developing countries.

At the global level, a large number of new candidate varieties are being generated for testing every year, thus underlining the need for establishing their clear-cut diagnostic features. Certain diagnostic features for released crop varieties are generally known and followed in seed certification procedures, accurate identification keys, giving detailed description on a comparative basis with clearcut features of distinctness are in general, lacking; and thus cases of confusion in seed certification and quality control arise.

\section{Materials and Methods}

Twenty five forage sorghum genotypes Bmr1, Bmr-2, Bmr-3, Bmr-4, Bmr-5, COFS 29, HJ 513, HC 260, IS 2205, IS 2389, DUGGI, CSV 21F, S 490, S 437, S 651, G 46, SSG 593, HC 136, HC 171, HJ 541, PC 5, PC 7, PC 8, ICSV 700, HC 308 were used in present study. The material was collected from Forage Section, Department of Genetics and Plant Breeding CCSHAU, Hisar and sowing was done on July 4, 2015. The field experiment was conducted at Research Area of Forage Section, Department of Genetics and Plant Breeding, CCS Haryana Agricultural University, Hisar. Observations were recorded on 31 quantitative variables.

\section{Results and Discussion}

\section{Classification on the basis of morphological characteristics}

Sorghum genotypes were grouped into different classes on the basis of observation recorded as per guideline of Protection of Plant Varieties \& Farmers Right Authority for morphological characters in the field at different stages of plant growth.

\section{Seedling Anthocyanin Colouration of Coleoptiles}

The classification of different genotypes on the basis of seedling anthocyanin colouration of coleoptiles was presented in Table 2.1. Nine genotypes were grouped under yellow green and remaining sixteen genotypes were under greyed purple.

\section{Leaf Sheath Anthocyanin Colouration}

On the basis of leaf sheath anthocyanin colouration genotypes were classified into two categories. Eleven genotypes were yellow green and remaining fourteen genotypes were greyed purple in colour. The classification of genotypes has been presented in Table 2.2.

\section{Leaf Midrib Colour $\left(5^{\text {th }}\right.$ fully developed leaf)}

Genotypes were classified on the basis of leaf midrib colour of $5^{\text {th }}$ fully developed leaf in to four groups. Eight genotypes were white, eleven genotypes were having yellow green, two were grey yellow and four were grey purple in their leaf midrib colour. Brown midrib genotypes were either grey purple or grey yellow. The classifications of genotypes were represented in Table 2.3.

Time to Panicle Emergence $(50 \%$ of the plants with $50 \%$ of anthesis)

On the basis of time of panicle emergence genotypes were classified mainly in to four categories. Seven genotypes were early (>65days), SSG 59-3 was medium (66-75 days), eight genotypes were late (76-85 days) and remaining eight genotypes were very late (> 85 days) for panicle emergence.

All brown midrib genotypes were early. The classifications of genotypes has been presented in Table: 2.4 . 


\section{Height of the plant up to the Base of flag Leaf}

Classification of genotypes on the basis of plant height up to the base of flag leaf has been presented in Table 2.5.Thirteen genotypes were tall, eight genotypes were having medium and four genotypes had short height and it was interesting to note that four out of five brown midrib genotypes were short and remaining one was medium.

\section{Flag leaf yellow colouration of midrib}

On the basis of flag leaf yellow colouration of midrib genotypes were classified into two categories. Fifteen genotypes were with white mid rib and remaining genotypes were with yellow green midrib colour. In brown midrib genotypes, yellow colouration was absent. The classification of genotypes has been presented in Table 2.6.

\section{Lemma Arista Formation}

Twenty five genotypes were classified on the basis of lemma arista formation were grouped into two categories. Nineteen had presence of lemma arista and in remaining had absent lemma arista was absent.

In all five brown midrib lines lemma arista was absent. The classification of genotypes has been presented in Table: 2.7.

\section{Stigma Anthocyanin Colouration}

On the basis of stigma anthocyanin colouration genotypes were classified into two categories.

Ten genotypes were without stigma anthocyanin colouration and remaining fifteen genotypes had stigma anthocyanin colouration. The classification of genotypes has been presented in Table 2.8.

\section{Stigma Yellow Colouration}

Classification of genotypes on the basis of stigma yellow colouration had been presented in Table 2.9. Fourteen genotypes were without yellow colouration and remaining eleven genotypes were having yellow colouration in their stigma.

\section{Stigma Length}

On the basis of stigma length genotypes were grouped into two groups. Fourteen genotypes were having $>2 \mathrm{~mm}$ stigma length and remaining eleven had 1-2 mm stigma length. All brown midrib genotypes had medium stigma length i.e. (1-2 mm). The classification of genotypes was represented in Table 2.10.

\section{Flower with Pedicel Length of Flower}

On the basis of flower with pedicel length of flower genotypes were grouped into three groups. Six genotypes were medium, 10 genotypes were with long pedicel and remaining nine genotypes were with very long pedicel length. Brown midrib genotypes had either medium or long pedicel length. The classification of genotypes has been presented in Table 2.11.

\section{Anther Length}

On the basis of anther length genotypes were classified into two categories. Four Genotypes had medium anther length and remaining twenty one genotypes had short anther length.

Four brown midrib genotypes i.e. BMR-1, BMR-2, BMR-3 and BMR-5 has short anther length whereas fifth genotytpe i.e. BMR-4 had medium anther length.

The classification of genotypes on the basis of anther length has been presented in Table 2.12 . 


\section{Anther Colour of Dry Anther}

On the basis of anther colour of dry anther genotypes were categerized into four groups. Twelve genotypes were yellow orange, four genotypes were orange, five genotypes were orange red and remaining four genotypes were with grayed orange anther colour. All brown midrib genotypes had orange red anther color of dry anther except BMR-4 which had yellow orange color of dry anthers. The classification of genotypes was represented in Table 2.13.

\section{Panicle Length of Branches}

Classification of genotypes on the basis of panicle length of branches had been presented in Table 2.14. Genotypes were classified into four categories; genotypes BMR-2, BMR-3 and $\mathrm{HC} 136$ had short $(<5.1 \mathrm{~cm})$; fourteen genotypes had medium $(5.1-10 \mathrm{~cm})$; five genotypes had long $(10.1-15 \mathrm{~cm})$ and genotypes COFS 29, ICSV 700 and SSG 59-3 had very long panicle length of branches.

\section{Panicle Density at Maturity}

On the basis of panicle density genotypes were classified into five categories. Two genotypes had very loose; four genotypes had loose; eight genotypes had semi loose; nine genotypes had semi compact and genotypes HC136 and BMR-2 had compact in their panicle density. The classifications of genotypes have been presented in Table 2.15.

\section{Panicle Shape}

Genotypes classified on the basis of panicle shape have been presented in Table 2.13.

Nine genotypes had symmetrical panicle; seven had panicle broader in lower part and seven genotypes had pyramidal in their panicle shape and BMR-5 and S651 had panicle broader in upper part.

\section{Neck of Panicle Visible above Sheath}

On the basis of neck of panicle visible above sheath genotypes were classified into four categories.

Seven genotypes had very short $(<5.1 \mathrm{~cm})$; eight genotypes had short $(5.1-10 \mathrm{~cm})$; five genotypes had medium $(10.1-15 \mathrm{~cm})$ and five genotypes had long $(15.1-20 \mathrm{~cm})$ neck of panicle visible above sheath. The classification of genotypes has been presented in Table 2.17.

\section{Glume Length}

Genotypes classified on the basis of glume length have been presented in Table 2.18.Two genotypes had very short; seven genotypes had short; ten genotypes had medium; three genotypes had long and three genotypes had very long glume.

\section{Caryopsis Colour}

On the basis of caryopsis colour genotypes were classified into four categories. Two genotypes had white; fifteen genotypes had yellow white; two genotypes had yellow orange and remaining six genotypes had grayed orange caryopsis color. The classification of genotypes has been presented in Table 2.19.

\section{Grain Shape in Dorsal view}

On the basis of grain shape in dorsal view classification of genotypes has been presented in Table 2.20. Genotypes were divided into three categories; thirteen genotypes were circular; seven genotypes were elliptic and remaining five genotypes were narrow elliptic in their grain shape in case of dorsal view. All brown midrib genotypes had circular grain shape except BMR-5 which has narrow elliptic shape. 
Table.1 1Morphological characters as per DUS parameters

\begin{tabular}{|c|c|c|c|}
\hline & Parameters & States & Stage of observation \\
\hline 1.1 & $\begin{array}{l}\text { Seedling : Anthocyanin } \\
\text { colouration of coleoptiles }\end{array}$ & $\begin{array}{l}\text { Yellow green } \\
\text { Greyed purple }\end{array}$ & $\begin{array}{c}\text { Seedling } \\
7-8 \text { days after sowing }\end{array}$ \\
\hline 1.2 & $\begin{array}{l}\text { Leaf sheath : Anthocyanin } \\
\text { colouration }\end{array}$ & $\begin{array}{l}\text { Yellow green } \\
\text { Greyed purple }\end{array}$ & $5^{\text {th }}$ leaf \\
\hline 1.3 & $\begin{array}{l}\text { Leaf : Midrib colour } \\
\left(5^{\text {th }} \text { fully developed leaf }\right)\end{array}$ & $\begin{array}{c}\text { White } \\
\text { Yellow green } \\
\text { Greyed yellow } \\
\text { Greyed purple }\end{array}$ & $5^{\text {th }}$ leaf \\
\hline 1.4 & $\begin{array}{l}\text { Plant : Time of panicle } \\
\text { emergence (50\% of the plants } \\
\text { with } 50 \% \text { anthesis) }\end{array}$ & $\begin{array}{c}\text { Very early ( }<56 \text { days) } \\
\text { Early }(56-65 \text { days }) \\
\text { Medium ( } 66-75 \text { days) } \\
\text { Late }(76-85 \text { days }) \\
\text { Very late }(>85 \text { days })\end{array}$ & Panicle emergence \\
\hline 1.5 & $\begin{array}{l}\text { Plant : Natural height of plant } \\
\text { upto base of flag leaf }\end{array}$ & $\begin{array}{c}\text { Very short }(<76 \mathrm{~cm}) \\
\text { Short }(76-150 \mathrm{~cm}) \\
\text { Medium }(151-225 \mathrm{~cm}) \\
\text { Tall }(226-300 \mathrm{~cm}) \\
\text { Very tall }(>300 \mathrm{~cm})\end{array}$ & Panicle emergence \\
\hline 1.6 & $\begin{array}{c}\text { Flag leaf : Yellow colouration } \\
\text { of midrib }\end{array}$ & $\begin{array}{l}\text { Absent } \\
\text { Present }\end{array}$ & Panicle emergence \\
\hline 1.7 & Lemma : Arista formation & $\begin{array}{l}\text { Absent } \\
\text { Present }\end{array}$ & Flowering \\
\hline 1.8 & $\begin{array}{l}\text { Stigma : Anthocyanin } \\
\text { colouration }\end{array}$ & $\begin{array}{l}\text { Absent } \\
\text { Present }\end{array}$ & $\begin{array}{l}\text { Upper portion of the } \\
\text { panicle at the end of } \\
\text { flowering }\end{array}$ \\
\hline 1.9 & Stigma : Yellow colouration & $\begin{array}{l}\text { Absent } \\
\text { Present }\end{array}$ & Flowering \\
\hline 1.10 & Stigma : Length & $\begin{array}{l}\text { Short }(<1 \mathrm{~mm}) \\
\text { Medium }(1-2 \mathrm{~mm}) \\
\text { Long }(>2 \mathrm{~mm})\end{array}$ & Flowering \\
\hline 1.11 & $\begin{array}{c}\text { Flower with pedicel : Length } \\
\text { of flower }\end{array}$ & $\begin{array}{l}\text { Very short } \\
\text { Short } \\
\text { Medium } \\
\text { Long } \\
\text { Very long }\end{array}$ & Flowering \\
\hline 1.12 & Anther : Length & $\begin{array}{l}\text { Short }(<3 \mathrm{~mm}) \\
\text { Medium }(3-4 \mathrm{~mm}) \\
\text { Long }(>4 \mathrm{~mm})\end{array}$ & Flowering \\
\hline 1.13 & Anther : Colour of dry anther & $\begin{array}{l}\text { Yellow orange } \\
\text { Orange } \\
\text { Orange red } \\
\text { Grayed orange }\end{array}$ & $\begin{array}{l}\text { End of flowering } \\
\text { Flowering }\end{array}$ \\
\hline
\end{tabular}




\begin{tabular}{|c|c|c|c|}
\hline 1.14 & Number of leaves per plant & $\begin{array}{c}\text { Less }(<15) \\
\text { Medium }(15-40) \\
\text { More }(>40)\end{array}$ & $\begin{array}{l}\text { Physiological } \\
\text { maturity }\end{array}$ \\
\hline 1.15 & Stem girth $(\mathrm{cm})$ & $\begin{array}{c}\text { Small }(<2 \mathrm{~cm}) \\
\text { Medium }(2-4 \mathrm{~cm}) \\
\text { Large }(>4 \mathrm{~cm})\end{array}$ & $\begin{array}{l}\text { Physiological } \\
\text { maturity }\end{array}$ \\
\hline 1.16 & Number of tillers per plant & $\begin{array}{l}\text { Less }(<2) \\
\text { Medium }(2-5) \\
\text { More }(>5)\end{array}$ & $\begin{array}{l}\text { Physiological } \\
\text { maturity }\end{array}$ \\
\hline 1.17 & $\begin{array}{l}\text { Panicle : Length of branches } \\
\text { (middle third of panicle) }\end{array}$ & $\begin{array}{c}\text { Short }(<5.1 \mathrm{~cm}) \\
\text { Medium }(5.1-10 \mathrm{~cm}) \\
\text { Long }(10.1-15 \mathrm{~cm}) \\
\text { Very long }(>15 \mathrm{~cm})\end{array}$ & $\begin{array}{l}\text { Physiological } \\
\text { maturity }\end{array}$ \\
\hline 1.18 & $\begin{array}{l}\text { Panicle : Density at maturity } \\
\text { (ear head compactness) }\end{array}$ & $\begin{array}{l}\text { Very loose } \\
\text { Loose } \\
\text { Semi loose } \\
\text { Semi compact } \\
\text { Compact }\end{array}$ & $\begin{array}{l}\text { Physiological } \\
\text { maturity }\end{array}$ \\
\hline 1.19 & Panicle : Shape & $\begin{array}{c}\text { Reversed pyramid } \\
\text { Panicle broader in upper part } \\
\text { Symmetric } \\
\text { Panicle broader in lower part } \\
\text { Pyramidal }\end{array}$ & $\begin{array}{l}\text { Physiological } \\
\text { maturity }\end{array}$ \\
\hline 1.20 & $\begin{array}{l}\text { Neck of panicle : Visible } \\
\text { length above sheath }\end{array}$ & $\begin{array}{l}\text { Absent or very short }(<5.1 \mathrm{~cm}) \\
\text { Short }(5.1-10 \mathrm{~cm}) \\
\text { Medium }(10.1-15 \mathrm{~cm}) \\
\text { Long }(15.1-20 \mathrm{~cm}) \\
\text { very long }(>20 \mathrm{~cm})\end{array}$ & $\begin{array}{l}\text { Physiological } \\
\text { maturity }\end{array}$ \\
\hline 1.21 & Glume : Length & $\begin{array}{l}\text { Very short ( } 25 \% \text { of grain covered) } \\
\text { Short (50\% of grain covered) } \\
\text { Medium ( } 75 \% \text { of grain covered) } \\
\text { Long ( } 100 \% \text { of grain covered) } \\
\text { Very long (longer than the grain) }\end{array}$ & $\begin{array}{l}\text { Physiological } \\
\text { maturity }\end{array}$ \\
\hline 1.22 & Grain : Threshability & $\begin{array}{l}\text { Freely threshable } \\
\text { (<11\% unthreshed grain) } \\
\text { Partly thresahble } \\
\text { (11 - 50\% unthreshed grain) } \\
\text { Difficult to thresh } \\
\text { ( }>50 \% \text { unthreshed grain) }\end{array}$ & Maturity \\
\hline 1.23 & $\begin{array}{l}\text { Caryopsis : Colour after } \\
\text { threshing }\end{array}$ & $\begin{array}{l}\text { White RHS } 155 \\
\text { Greyed white RHS } 156 \\
\text { Yellow white RHS } 158 \\
\text { Yellow orange RHS 14-20 } \\
\text { Greyed orange RHS } 200\end{array}$ & After threshing \\
\hline 1.24 & Grain : Shape (in dorsal view) & $\begin{array}{l}\text { Narrow elliptic } \\
\text { Elliptic }\end{array}$ & After threshing \\
\hline
\end{tabular}




\begin{tabular}{|c|c|c|c|}
\hline & & Circular & \\
\hline 1.25 & Grain : Shape in profile view & $\begin{array}{l}\text { Narrow elliptic } \\
\text { Elliptic } \\
\text { Circular }\end{array}$ & After threshing \\
\hline 1.26 & Grain : Size of mark of germ & $\begin{array}{l}\text { Very small } \\
\text { Small } \\
\text { Medium } \\
\text { Large } \\
\text { Very large }\end{array}$ & After threshing \\
\hline 1.27 & $\begin{array}{l}\text { Grain : Texture of endosperm } \\
\quad \text { (in longitudinal section) }\end{array}$ & $\begin{array}{l}\text { Fully vitreous ( } 100 \% \text { corneous) } \\
3 / 4 \text { vitreous ( } 75 \% \text { corneous) } \\
\text { Half vitreous ( } 50 \% \text { corneous) } \\
3 / 4 \text { farinaceous ( } 25 \% \text { corneous) } \\
\text { Fully farinaceous ( } 0 \% \text { corneous) }\end{array}$ & After threshing \\
\hline 1.28 & $\begin{array}{c}\text { Grain : Colour of vitreous } \\
\text { albumen }\end{array}$ & $\begin{array}{c}\text { Greyed yellow RHS 160-162 } \\
\text { Greyed orange RHS } 166 \\
\text { Greyed purple RHS N } 187\end{array}$ & After threshing \\
\hline 1.29 & Grain : Luster & $\begin{array}{l}\text { Non-lustrous } \\
\text { Lustrous }\end{array}$ & After threshing \\
\hline 1.30 & Green fodder yield/plant & & After harvesting \\
\hline 1.31 & Dry fodder yield/plant & & After harvesting \\
\hline
\end{tabular}

Table.2 Classification of Sorghum genotypes on the basis of seedling anthocyanin colouration of colieoptiles

\begin{tabular}{|l|l|}
\hline Yellow Green(9) & $\begin{array}{l}\text { BMR-1, BMR-2, BMR-3, HJ 513, HC260, HC136, PC7, HC 171, } \\
\text { HJ541 }\end{array}$ \\
\hline Grayed Purple (16) & $\begin{array}{l}\text { BMR-4, BMR-5, PC5, PC8, COFS29, IS2205, IS2389, DUGGI, } \\
\text { CSV21F, S490, S437, S651, G46, SSG59-3, ICSV700, HC308. }\end{array}$
\end{tabular}

Table.3 Classification of sorghum genotypes on the basis of leaf sheath anthocyanin colouration

\begin{tabular}{l|l} 
Yellow Green(11) & BMR-1, BMR-4, BMR-3, BMR-5, HJ513, HC260, HC136, HJ541, \\
HC171, PC7, ICSV700 & Grayed Purple (14) \\
& $\begin{array}{l}\text { BMR-2, COFS29, IS 2205, IS 2389, DUGGI, CSV21F, S490, S437, } \\
\text { S651, G46, SSG59-3, PC5, PC8, HC308 }\end{array}$
\end{tabular}

Table.4 Classification of Sorghum genotypes on the basis of leaf midrib colour of $5^{\text {th }}$ fully developed leaf

\begin{tabular}{|l|l|}
\hline White(8) & COFS29, HJ513, HC260, IS2205, IS2389, DUGGI, S490, S651 \\
\hline Yellow green(11) & $\begin{array}{l}\text { CSV21F, PC5, S437, SSG59-3, HC136, HC171, ICSV700, HJ541, } \\
\text { PC8,G46,HC308 }\end{array}$ \\
\hline Grey yellow(2) & BMR-3, PC7 \\
\hline Grey purple (4) & BMR-1, BMR-2, BMR-5,BMR-4
\end{tabular}


Table.5 Classifications of Sorghum genotypes on the basis of time of panicle emergence (50\% of the plants with $50 \%$ anthesis)

\begin{tabular}{|l|l|}
\hline Early (<65days) (7) & BMR-1, BMR-2, BMR-3, BMR-4, BMR-5, HC260, ICSV700 \\
\hline Medium (66-75 days) (2) & DUGGI, SSG59-3 \\
\hline Late (76-85 days) (8) & HC308, PC5, PC7, IS2205, IS2389, CSV21F, S490, S651 \\
\hline Very late (>85 days) (8) & C0FS29, HJ513, HJ541, S437, PC8, G46, HC136, HC171 \\
\hline
\end{tabular}

Table.6 Classification of sorghum genotypes on the basis of height of plant up to the base of flag leaf

\begin{tabular}{l|l}
\hline Short $(76-150 \mathrm{~cm})(4)$ & BMR-1, BMR-3, BMR-4, BMR-5 \\
\hline Medium $(151-255 \mathrm{~cm})(8)$ & BMR-2, HC260, DUGGI, ICSV700, S651, PC5, PC7, PC8 \\
\hline Tall $(226-300 \mathrm{~cm})(13)$ & $\begin{array}{l}\text { IS2389, S490, COFS29, HJ513, IS2205, S437, G46, SSG59-3, } \\
\text { HC136, HC171, HJ541, HC308, CSV21F }\end{array}$
\end{tabular}

Table.7 Classification of Sorghum genotypes on the basis of flag leaf yellow colouration of midrib

\begin{tabular}{|l|l|}
\hline Absent (15) & BMR-1, BMR-2, BMR-3, BMR-4, BMR-5, COFS29, HJ513, HC260, IS2205, \\
& G46, SSG59-3, PC5, PC7, PC8, ICSV700 \\
\hline Present (10) & IS2389, HC136, HC171, HJ541, DUGGI, CSV21F, S490, S437, S651, HC308
\end{tabular}

Table.8 Classification of Sorghum genotypes on the basis of lemma arista formation

\begin{tabular}{l|l} 
Present (19) & $\begin{array}{l}\text { BMR-1, BMR-3, BMR-5, COFS29, S490, S437, S651, G46, SSG59-3, HC136, } \\
\text { HC171, HJ541, PC7, IS2205, IS2389, CSV21F, ICSV700, HC308, HJ513 }\end{array}$ \\
\hline Absent (6) & BMR-2, BMR-4, HC260, DUGGI, PC5, PC8
\end{tabular}

Table.9 Classification of Sorghum genotypes on the basis of stigma anthocyanin colouration

\begin{tabular}{|l|l|}
\hline Absent (10) & $\begin{array}{l}\text { BMR-2, BMR-4, IS2205, DUGGI, G46, HC136, HC171, PC5, PC 8, ICSV } \\
700\end{array}$ \\
\hline Present(15) & $\begin{array}{l}\text { BMR-1, BMR-3, BMR-5, COFS29, HJ513, HC260, IS2389, CSV21F, } \\
\text { S490,S437, S651, SSG59-3, HJ541, PC7, HC308 }\end{array}$ \\
\hline
\end{tabular}

Table.10 Classification of Sorghum genotypes on the basis of stigma yellow colouration

\begin{tabular}{|l|l|}
\hline Absent (14) & $\begin{array}{l}\text { BMR-2, BMR-3, BMR-5, HJ 513, HC260, IS2205, S437, S651, G46, HC136, } \\
\text { HC171, HJ541, PC5, ICSV700 }\end{array}$ \\
\hline Present (11) & $\begin{array}{l}\text { BMR-1, BMR-4, COFS29, IS2389, DUGGI, CSV21F, S490, SSG59-3, PC7, } \\
\text { PC8, HC308 }\end{array}$
\end{tabular}


Table.11 Classification of Sorghum genotypes on the basis of stigma length

\begin{tabular}{l|l}
\hline Medium(1-2 mm) (11) & $\begin{array}{l}\text { BMR-1, BMR-2, BMR-3, BMR-4, BMR-5, HC260, DUGGI, } \\
\text { CSV21F, S651, PC8 ICSV700 }\end{array}$ \\
\hline Long (>2 mm) (14) & $\begin{array}{l}\text { COFS29, HJ513, IS2205, IS2389, S490, S437, G46, SSG59-3, } \\
\text { HC136, HC171, HJ541, PC5, PC7, HC308 }\end{array}$
\end{tabular}

Table.12 Classification of Sorghum genotypes on the basis of flower with pedicel length of flower

\begin{tabular}{|l|l|}
\hline Medium (6) & BMR-2, BMR-3, HJ513, HC260, DUGGI, S651 \\
\hline Long (10) & BMR-1, BMR-4, BMR-5, S490, S437, HC136, HC171, HJ541, PC7, HC308 \\
\hline Verylong (9) & COFS29, IS2205, IS2389, PC8, CSV21F, G46, SSG59-3, PC5, ICSV700 \\
\hline
\end{tabular}

Table.13 Classification of Sorghum genotypes on the basis of anther length

\begin{tabular}{l|l} 
Short $(<3 \mathrm{~mm})(21)$ & $\begin{array}{l}\text { HC260, DUGGI, S437, HC136, HC171, PC5, PC7, BMR-2, BMR-3, } \\
\text { BMR-5, ICSV700, HC308, S490, IS2389, COFS29, HJ513, SSG59-3, } \\
\text { HJ541, BMR-1, S651, PC8 }\end{array}$ \\
\hline Medium (3-4 mm) (4) & BMR-4, IS2205, CSV21F, G46
\end{tabular}

Table.14 Classification of Sorghum genotypes on the basis of anther colour of dry anther

\begin{tabular}{|l|l|}
\hline Yellow orange (12) & $\begin{array}{l}\text { BMR-4, HC260, IS2205, DUGGI, S437, G46, HC136, HC171, PC5, } \\
\text { PC7, HJ541, PC8 }\end{array}$ \\
\hline Orange (4) & ICSV700, HC308, S490, IS2389 \\
\hline Orange red (5) & BMR-2, BMR-3, BMR-5, BMR-1, S651 \\
\hline Grayed orange (4) & COFS29, HJ513, CSV21F, SSG59-3
\end{tabular}

Table.15 Classification of Sorghum genotypes on the basis of panicle length of branches

\begin{tabular}{|l|l|}
\hline Short $(<5.1 \mathrm{~cm})(3)$ & HC136, BMR-2, BMR-3 \\
\hline Medium $(5.1-10 \mathrm{~cm})(14)$ & $\begin{array}{l}\text { S437, HC308, HJ513, IS2205, S490, HJ541, HC171, BMR-1, } \\
\text { BMR-4, BMR-5,CSV21F, PC5, PC7, PC8 }\end{array}$ \\
\hline Long $(10.1-15 \mathrm{~cm})(5)$ & G46, IS2389, HC260, DUGGI, S651 \\
\hline Very long $(>15 \mathrm{~cm})(3)$ & COFS 29, SSG59-3, ICSV700
\end{tabular}

Table.16 Classification of genotypes on the basis of panicle density at maturity

\begin{tabular}{|l|l|}
\hline Very loose (2) & SSG59-3, COFS29 \\
\hline Loose (4) & DUGGI, IS2389, CSV21F, G46, \\
\hline Semi loose (8) & BMR-3, HJ513, IS2205, S490, S437, S651, HJ541, HC171 \\
\hline Semi compact (9) & PC7, ICSV700, HC260, BMR-1, BMR-5, PC5, PC8, HC308, BMR-4 \\
\hline Compact (2) & BMR-2, HC136 \\
\hline
\end{tabular}


Table.17 Classification of sorghum genotypes on the basis of panicle shape

\begin{tabular}{|l|l|}
\hline Symmetrical (9) & $\begin{array}{l}\text { BMR-1, BMR-2, BMR-3, S490, HJ541, PC5, PC8, } \\
\text { HC308, CSV21F }\end{array}$ \\
\hline Panicle broader in upper part (2) & BMR-5, S651 \\
\hline Panicle broader in lower part (7) & \begin{tabular}{l} 
BMR-4, HJ513, HC260, IS2205, S437, HC136, PC7 \\
\hline Pyramidal (7)
\end{tabular} \\
$\begin{array}{l}\text { COFS29, IS2389, DUGGI, G46, SSG59-3, HC171, } \\
\text { ICSV700 }\end{array}$
\end{tabular}

Table.18 Classification of genotypes on the basis of neck of panicle visible above sheath

\begin{tabular}{|l|l|}
\hline Absent or very short $(<5.1 \mathrm{~cm})(7)$ & BMR-1, DUGGI, S490, S437, HJ541, PC8, HC308 \\
\hline Short $(5.1-10 \mathrm{~cm})(8)$ & $\begin{array}{l}\text { BMR-2, HC136, PC5, BMR-4, BMR-5, IS2205, } \\
\text { CSV21F, PC7 }\end{array}$ \\
\hline Medium $(10.1-15 \mathrm{~cm})(5)$ & BMR-3, HC171, HJ513, S651, G46 \\
\hline Long $(15.1-20 \mathrm{~cm})(5)$ & SSG59-3, HC260, IS 2389, COFS 29, ICSV 700
\end{tabular}

Table.19 Classification of Sorghum genotypes on the basis of glume length

\begin{tabular}{|l|l|}
\hline Very short (25\% of grain covered)(2) & BMR-1, BMR-4 \\
\hline Short (50\% of grain covered) (7) & BMR-2, HJ 513, S 490, HC 136, G 46, HJ 541, PC 5 \\
\hline Medium (75\% of grain covered) (10) & BMR-3, BMR-5, HC260, IS2389, IS2205, HC308, \\
\hline Long (100\% of grain covered) (3) & PC8HC171, S437,CSV21F \\
\hline Very long (longer than the grain) (3) & COFS29, DUG-3, S651 \\
\hline
\end{tabular}

Table.20 Classification of genotypes on the basis of caryopsis colour

\begin{tabular}{|l|l}
\hline White (2) & IS 2205, S 490 \\
\hline Yellow white (15) & $\begin{array}{l}\text { BMR-1, BMR-3, BMR-4, PC5, PC7, PC8, HC308, S651, HC136, } \\
\text { HC171, BMR-5, HJ513, HC260, S437, HJ541 }\end{array}$ \\
\hline Yellow orange (2) & BMR-2, CSV21F \\
\hline Grayed orange (6) & COFS29, DUGGI, IS2389, G46, SSG59-3, ICSV700
\end{tabular}

Table.21 Classification of Sorghum genotypes on the basis of Grain shape in dorsal view

\begin{tabular}{|l|l|}
\hline Circular (13) & $\begin{array}{l}\text { BMR-1, BMR-2, BMR-3, BMR-4, IS2205, S490, HC260, PC7, PC8, } \\
\text { HC136, PC5, ICSV700, CSV21F }\end{array}$ \\
\hline Elliptic (7) & IS2389, S437, HC308, HJ513, S651, HC171, HJ541 \\
\hline Narrow elliptic (5) & COFS29, DUGGI, G46, SSG59-3, BMR-5
\end{tabular}


Table.22 Classification of Sorghum genotypes on the basis of threshability

Freely threshable

(<11\% unthreshed grain) (6)

Partly threshable $(11-50 \%$

unthreshed grain) (14)

Difficult to thresh

(> 50\% unthreshed grain) (5)
BMR-4, BMR-5, BMR-2, HJ513, DUGGI, PC5

BMR-1, BMR-3, HC260, HC136, HC171, HJ541, IS2389, PC7, PC8, IS2205, CSV21F, S490, G46, ICSV700 COFS29, S437, S651, SSG59-3, HC308

Table.23 Classification of Sorghum genotypes on the basis of grain shape in profile view

\begin{tabular}{|l|l|}
\hline Circular (7) & BMR-3, HJ513, IS2205, S490, HC136, PC5, ICSV700 \\
\hline Elliptic (14) & BMR-1, BMR-2, BMR-4, BMR-5, HC260, IS2389, CSV21F, S437, \\
& S651, HC171, HJ541, PC7, PC8, HC308 \\
\hline Narrow elliptic (4) & COFS 29, DUGGI, G 46, SSG 59-3
\end{tabular}

Table.24 Classification of Sorghum genotypes on the basis of grain size of mark germ

\begin{tabular}{|l|l|}
\hline Small (4) & HC260, DUGGI, S651, PC8 \\
\hline Medium (10) & BMR-2, BMR-4, BMR-5, CSV 21F, S 490, S 437, HJ 541, PC 5, PC 7, HC 308 \\
\hline Large (11) & BMR-1, BMR-3, COFS29, HJ513, IS2205, IS2389, G46, SSG59-3, HC136, \\
& HC 171, ICSV 700
\end{tabular}

Table.25 Classification of Sorghum genotypes on the basis of grain texture of endosperm

\begin{tabular}{|l|l|}
\hline Vitreous (1) & COFS29 \\
\hline 3/4 Vitreous (2) & BMR-3, IS2205 \\
\hline 1/2 Vitreous (15) & $\begin{array}{l}\text { BMR-2, BMR-4, BMR-5, HJ513, HC260, IS2389, CSV21F, S490, } \\
\text { S437, S651, SSG59-3, PC5, PC7, PC8, ICSV700 }\end{array}$ \\
\hline 3/4 Farinacus (7) & BMR-1, DUGGI, G46, HC136, HC171, HJ541, HC308 \\
\hline
\end{tabular}

Table.26 Classification of Sorghum genotypes on the basis of grain colour of vitreous albumen

\begin{tabular}{l|l}
\hline Grayed Yellow (16) & $\begin{array}{l}\text { BMR-1, BMR-3, BMR-4, BMR-5, HJ513, HC308, S490, PC5, PC7, } \\
\text { HC136, HC171, HJ541, HC260, IS2205, DUGGI, CSV21F }\end{array}$ \\
\hline Grayed Orange (7) & BMR-2, S437, S651, G46, SSG59-3, PC8, ICSV700 \\
\hline Grayed Purple (2) & COFS29, IS2389
\end{tabular}

Table.27 Classification of sorghum genotypes on the basis of grain luster

\begin{tabular}{|l|l|}
\hline Lusterous (11) & $\begin{array}{l}\text { BMR-4, BMR-5, HJ513, HC260, IS2205, S490, S437, HC171, HJ541, } \\
\text { PC5, HC308 }\end{array}$ \\
\hline Non Lusterous (14) & $\begin{array}{l}\text { BMR-1, BMR-2, BMR-3, COFS29, IS2389, DUGGI, ICSV700, PC8, } \\
\text { CSV21F, S651, G46, SSG59-3, HC136, PC7 }\end{array}$
\end{tabular}


Table.28 Promising genotypes on the basis of quality parameters

\begin{tabular}{|c|l|l|l|}
\hline S. No. & Character & Range & Promising genotype \\
\hline $\mathbf{1}$ & Crude Protein (\%) & $8.2-9.3$ & $\begin{array}{l}\text { (Higher value) BMR-2 (9.3), HC 171 (9.3), ICSV 700 } \\
\text { (9.3), S 437 (9.2), SSG 59-3 (9.2) }\end{array}$ \\
\hline $\mathbf{2}$ & IVDMD (\%) & $59.4-68.2$ & $\begin{array}{l}\text { (Higher value) } \\
\text { BMR-3 (68.2), CSV 21F (68.2), S 490 (68.1), S 651 } \\
(67.2), \text { PC 5 (66.2) }\end{array}$ \\
\hline $\mathbf{3}$ & NDF (\%) & $61.2-75.9$ & $\begin{array}{l}\text { (Lower value) PC 7 (61.2), G 46 (64.1), IS 2205 } \\
\text { (65.3), BMR-1 (65.5), BMR-3 (65.6) }\end{array}$ \\
\hline $\mathbf{4}$ & ADF (\%) & $36.1-56.3$ & $\begin{array}{l}\text { (Lower value) BMR-2 (36.1), HC 260 (36.4), BMR- } \\
\text { (36.5), HJ 513 (37.5) }\end{array}$ \\
\hline $\mathbf{5}$ & Lignin (\%) & $2.6-5.8$ & $\begin{array}{l}\text { (Lower value) BMR-3 (2.6), BMR-2 (3.0), BMR-4 } \\
\text { (3.0), BMR-1 (3.1), PC 5 (3.2) }\end{array}$ \\
\hline $\mathbf{6}$ & $\begin{array}{l}\text { DDM (Digestible } \\
\text { dry matter) (q/ha) }\end{array}$ & $26.6-94.4$ & $\begin{array}{l}\text { (Higher value) CSV 21F (94.4), S 490 (90.4), ICSV } \\
\text { 700 (68.0), HJ 541 (64.1), S 651 (64.0) }\end{array}$ \\
\hline $\mathbf{7}$ & $\begin{array}{l}\text { Protein yield (q/ha) } \\
\text { (Higher value) CSV 21F (13), S 490 (11.9), ICSV 700 } \\
\text { (9.9), HC 308 (9.5), PC 5 (8.6) }\end{array}$ \\
\hline
\end{tabular}

\section{Threshability}

On the basis of threshability genotypes were classified into three categories. Six genotypes were freely threshable; fourteen genotypes were partly threshable and remaining five genotypes were difficult to thresh. The classification of genotypes has been presented in Table 2.21

\section{Grain shape in profile view}

On the basis of grain shape in profile view classification of genotypes has been presented in Table 2.22. Seven genotypes were circular; fourteen genotypes were elliptic; remaining four genotypes were narrow elliptic in their grain shape.

\section{Grain size of mark germ}

Genotypes classified in three categories on the basis of grain size of mark of germ have been presented in Table 2.23. Ten genotypes had medium; eleven genotypes had large and four genotypes had small grain size of mark germ.

\section{Grain texture of endosperm}

On the basis of grain texture of endosperm genotypes were classified into four categories. Genotype COFS29 had $100 \%$ vitreous endosperm; two genotypes had 3/4 vitreous; fifteen genotypes had $1 / 2$ vitreous and seven genotypes had $3 / 4$ farinaceous texture of endosperm. The classification of genotypes has been presented in Table 2.24.

\section{Grain colour of Vitreous albumen}

On the basis of grain colour of vitreous albumen genotypes were classified into three categories. Sixteen genotypes had greyed yellow; seven genotypes had greyed orange; and remaining two genotypes had greyed purple colour of vitreous albumen. The classification of genotypes has been presented in Table 2.25.

\section{Grain luster}

On the basis of grain luster genotypes were categories into two groups. Eleven genotypes were having lusterous grain and remaining 
fourteen genotypes were having non lusterous grain. The classifications of genotypes has been presented in Table 2.26.

\section{Classification on the basis of quality parameters}

Promising genotypes selected on the basis of quality are presented in Table 4.1 (b) depending on their utility i.e. higher and lower value.

\section{Morphological characteristic at Seedling stage}

A morphological characteristic of sorghum seedling anthocyanin colouraion of coleoptiles was examined at seedling stage for their characterization. Based on the variation observed on diagnostic character it was possible to distinguish all genotypes form each other. The difference provide the basis for characterizing the genotypes/lines broadly into two categories: greyed purple and yellow green. Nagaraja et al., (2000) characterized twenty three varieties including five hybrids of sorghum on the basis of seed colour and seedling morphology.

\section{$5^{\text {th }}$ leaf stage}

At $5^{\text {th }}$ leaf stage genotypes were characterized on the basis of two characters; leaf sheath anthocyanine colouration and leaf midrib colour of $5^{\text {th }}$ fully developed leaf. All genotypes were shown different colouration pattern as seedling anthocyanin colouration of coleoptiles. In case of leaf midrib colour difference provides the basis of characterizing the genotypes into four groups: white and yellow green, yellow green, grey purple. Sangwan et al., (2005) characterized 12 sorghum genotypes on the basis of midrib colour (white and green). Reddy et al., (2008) studied inheritance of midrib colour (brown and white) in 8 segregating populations.

\section{Panicle emergence stage}

Sorghum genotypes were characterized on the basis of time of panicle emergence $(50 \%$ of plants with $50 \%$ flowering), plant natural height of plant up to the base of flag leaf, and flag leaf yellow colouration of midrib at panicle emergence stage. Genotypes were grouped into three categories (medium, late and very late) for time of panicle emergence; whereas, genotypes were divided into two groups on the basis of plant height up to the base of flag leaf and genotypes were divided in two groups (present and absent) for flag leaf yellow colouration of midrib. Elangovan (2006) characterized 179 accessions of sorghum on the basis of days to $50 \%$ flowering along with other morphological characters. Reddy et al., (2009) studied divergence and genetic variability of 29 sorghum genotypes on the basis of time of panicle emergence. Reddy et al., (2008) studied inheritance pattern of midrib colour (recorded at, fifth leaf stage, sixth leaf stage and at the time of panicle emergence.

\section{Flowering stage}

Sorghum genotypes were classified into different groups based on lemma arista formation, stigma anthocyanine colouration, stigma yellow colouration, anther length, stigma length, colour of dry anther and flower with pedicle length of flower. Based on colour of dry anther genotypes were classified into three categories (yellow orange, orange and grayed orange) while, flower with pedicle length of flower also divided into three groups (medium, long and very long). However, stigma yellow colouration, stigma anthocyanine colouration anther length genotypes were divided into two groups respectively. In case of stigma length all genotypes were having long stigma along with lemma arista formation Reddy et al., (2006) characterized 269 designated lines of 
sorghum using DUS guidelines for sorghum. Reddy et al., (2008) studied the inheritance of awning in set of sorghum cultivars. Reddy et al., (2009) studied divergence and variability in 29 sorghum genotypes using 39 agro morphological traits using in DUS testing.

\section{Physiological maturity}

Nature of characteristics like, glume colour, plant height, stem diameter, panicle length of branches, panicle density at maturity, panicle shape, neck of panicle visible above sheath, glume length and grain threshability were recorded for characterization of genotypes at physiological maturity. On the basis of glume colour, neck of panicle visible above sheath and panicle density at maturity genotypes could be divided into five categories, respectively. In case of panicle shape and grain thershability genotypes were categories into three groups each. Whereas, plant height and stem diameter genotypes were categories in two groups, respectively. Pahuja et al., (2002) evaluated eighteen sorghum hybrids on the basis of plant height. Umakanth et al., (2002) studied diversity of forty eight sorghum genotypes on the basis of plant height and panicle length. Sangwan et al., (2005) studied identification of 12 sorghum genotypes on the basis of panicle compactness, shape of panicle and plant height. Elangovan et al., (2006) characterized 179 accessions on the basis of earhead compactness, earhead shape, glume colour, earhead length and plant height. Reddy et al., (2009) characterized 29 sorghum genotypes on the basis of plant height and panicle length.

\section{After threshing}

After threshing genotypes were classified on the basis of caryopsis colour, grain shape in dorsal view, grain shape in profile view, grain size of mark of germ, grain texture of endosperm (in longitudinal section), grain colour of vitreous albumen and grain luster. Genotypes were characterized in four groups on the basis of caryopsis colour and grain textures of endosperm. However, genotypes were divided in three groups on the basis of grain shape in dorsal view, grain shape in profile view, grain size of mark of germ and grain colour of vitreous albumen, respectively. Whereas, on the basis of grain luster genotypes were classified in two groups. Selvaraja et al., (2000) classified 19 sorghum varieties on the basis of length, breadth, length/breadth ratio and colour of seeds. Thangavel et al., (2005) classified 12 sorghum cultivars on the basis of seed colour, seed size, seed shape, size of mark of germ, texture of endosperm, colour of vitreous albumen and seed luster. Elangovan (2006) characterized 179 sorghum accessions on the basis of seed size and seed colour.

\section{References}

Elangovan, M. (2006) Characterization and evaluation of exotic sorghum germplasm collections. Int. J. Agric. Sci, 2(1), 231-234.

Nagaraja, G.S., Varier, A., Singh, U., Sharma, S.P. (2000) Characterization of inbreds and hybrids of sorghum \{Sorghum bicolor (L.) Moench\} by seed and seedling morphology and electrophoresis of seed proteins and isoenzymes. Plant Varieties and Seeds, 13(1), 17-29.

Pahuja, S.K., Grewal, R.P.S., Singh, N., Jindal, Y. and Pundir, S.R. (2002). Evaluation of forage sorghum hybrid for yield and morphological traits. Int. Sorghum Millets Newsletter. 43, 42-45.

Pholsen, S. and Suksri, A. (2007) Effect of phosphorus and potassium on growth, yield and fodder quality of IS 23585 forage sorghum cultivar (Sorghum 
bicolor (L.) Moench). Pakistan J. Bio. Sci, 10, 1604-1610.

Reddy, B.V.S., Sharma, H.C., Thakur, R.P., Ramesh, S. (2006) Special issue: Characterization of ICRISAT- bred Sorghum hybrid parents (set I). International Sorghum and Millets Newsletter, 47, 138.

Reddy, D.C.S, Audilakshmi, S. and Seetharam, N. (2009) Genetic variability and divergence for DUS testing traits in sorghum (Sorghum bicolor (L.) Moench). Indian J. Agric. Sci, 79(4), 286-290.

Reddy, R.N., Mohan, S.M., Madhusudhana, R., Umakantha, A.V., Saith, K. and Sriniwas, G. (2008) Inheritance of morphological characters in sorghum. $J$. SAT Agric. Res, 6, 1-3.
Sangwan, V.P., Chauhan, P., Ram, C. (2005) Varietal identification of forage sorghum through morphological characters. Seed Research, 33(1), 117118.

Selvaraju, P. and Sivasubramaniam, K. (2000) Identification keys for sorghum varieties. Madras Agricultural Journal, 87, 10-12.

Thangavel, P., Bhatati, A., Natarajan, N. and Evera, T. (2005) Varietal grouping in sorghum by seed and seedling morphology and response to chemical testing. Karnataka Journal of Agricultural Sciences, 18(3), 664-672.

Umakanth, A.V., Madhusudhana, R., Swarnlata, Kaul., Rana, B.S. (2002) Genetic diversity studies in sorghum. International Sorghum and Millets Newsletter, 43, 31-33.

\section{How to cite this article:}

Neha Rohila, Satyawan Arya, S. K. Pahuja, Pummy Kumari, Pinki, Kanika Rani and Nisha Devi. 2018. Morphological Characterization and Quality Parameters of Various Forage Sorghum Genotypes (Sorghum bicolor L. Moench). Int.J.Curr.Microbiol.App.Sci. 7(06): 20572071. doi: https://doi.org/10.20546/ijcmas.2018.706.243 